\title{
IL084:
}

\section{Analog Design-Assist CAD Tools in the Nanometer Era}

\author{
By \\ Mohamed Dessouky
}

\section{Abstract:}

With the advance of nanoscale fabrication processes, analog design is facing new challenges resulting from the associated complex processing. A new set of systematic mismatch effects appears due to layout. This includes shallow trench isolation stress, well-proximity and lithographic effects to name a few. The demand for new analog design-assist CAD tools is increasing. Such tools would simplify the task of the designer in order to achieve a successful analog and physical circuit design. The target is not full design automation, but rather design assistance on specific problems. In this paper, three directions are discussed; first, complex layout templates are introduced to simplify layout design, then electrical design for manufacturing (EDFM) is suggested to treat parametric yield issues arise from manufacturing process variation and layout issues, and finally automated circuit design migration is described to facilitate design porting needed for the accelerated pace of process scaling and time-to-market.

- Ain Shams University, Egypt 